\title{
Low-Profile Magnetic Loop Monopole Antenna Based on a Square Substrate-Integrated Cavity
}

\author{
Thomas Kaufmann and Christophe Fumeaux \\ School of Electrical and Electronic Engineering, The University of Adelaide, Adelaide, SA 5005, Australia \\ Correspondence should be addressed to Thomas Kaufmann; thomaska@eleceng.adelaide.edu.au
}

Received 15 October 2014; Accepted 26 December 2014

Academic Editor: Feifei Gao

Copyright ( 2015 T. Kaufmann and C. Fumeaux. This is an open access article distributed under the Creative Commons Attribution License, which permits unrestricted use, distribution, and reproduction in any medium, provided the original work is properly cited.

\begin{abstract}
A low-profile monopole antenna with height below $\lambda_{0} / 16$ is proposed. The antenna is based on a square substrate-integrated cavity radiating through apertures in its four side walls. This effectively creates a small square loop of magnetic currents, which radiates omnidirectionally as an electric monopole. The antenna cavity has a side length of less than $\lambda_{0} / 3$ and thus resonates in the monomode region, in a fundamental $T M_{11}$ mode. This means that the structure is robust in terms of feeding and manufacturing tolerances, as no parasitic modes can be excited. The designs of the cavity and the feed are introduced in detail. The measurement results from a manufactured prototype operating at $5.9 \mathrm{GHz}$ show good agreement with simulations and validate the proposed approach.
\end{abstract}

\section{Introduction}

An increasing number of technologies in the microwave region require low-profile monopole antennas. Emerging examples are in the dedicated short-range communications (DSRC) band for vehicle-to-vehicle (v2v) safety systems [1], which in the future might be integrated in automobile roofs. For these applications, an antenna backed by a ground plane that can be integrated into an RF substrate appears very attractive. A crucial requirement is an omnidirectional radiation pattern with maximum in or close to the substrate plane. Promising geometries to achieve these requirements can be based on low-profile resonant cavities, with fringing fields from thin apertures forming equivalent magnetic currents as described in detail later in this paper.

A selection of previous work on low-profile monopole antennas [2-8] is given in Table 1. Existing geometries can be roughly split into two categories: square and circular cavities, with corresponding $T M_{11}$ and higher-order modes for square patches and $T M_{01}$ and $T M_{02}$ modes for circular patches. The resonator height is generally influenced by the choice of cavity medium, and a number of these summarized examples use free-space cavities with considerable height $[2,6,7]$. In contrast, in order to achieve ultralow profile, promising realizations using substrate integration and vias have gained traction recently, for example, in $[9,10]$, supported by the low cost and high accuracy of automated printed circuit board manufacturing techniques.

The antenna presented in this paper is the first square patch monopole completely integrated in a substrate using shorting vias. Compared to previous work, this antenna combines compactness and low profile, while the proposed approach provides a new perspective to planar monopole antenna design: instead of being considered a loaded monopole, the structure is interpreted as an opened rectangular cavity, which offers a new design paradigm. In this framework, we present a symmetrical substrate-integrated cavity resonating with a fundamental $T M_{11}$ mode. The radiation originates from four slots along the side walls, which effectively creates a square magnetic current loop. This results in an equivalent electric monopole antenna that is

(i) radiating omnidirectionally,

(ii) compact (resonator side length $<\lambda_{0} / 3$ ),

(iii) low-profile (substrate height $<\lambda_{0} / 16$ ),

(iv) robust to tolerances (monomode operation). 
TABLE 1: Comparison of different low-profile monopole patch antennas from the literature.

\begin{tabular}{|c|c|c|c|c|c|c|c|c|}
\hline & This work & [2] & {$[3]$} & {$[4]$} & [5] & {$[6]$} & [7] & {$[8]$} \\
\hline Cavity geometry & $\begin{array}{c}\text { Square and } \\
\text { shorting vias }\end{array}$ & $\begin{array}{c}\text { Square and } 4 \\
\text { shorting strips }\end{array}$ & Circle & Circle & $\begin{array}{c}\text { Circle and } \\
\text { shorting vias }\end{array}$ & $\begin{array}{c}\text { Square and } 2 \\
\text { shorting strips }\end{array}$ & $\begin{array}{c}\text { Circle and } \\
\text { shorting strips }\end{array}$ & $\begin{array}{c}\text { Circle and } \\
\text { shorting vias }\end{array}$ \\
\hline Mode(s) & $T M_{11}$ & $T M_{11}, T M_{02}$ & $T M_{02}$ & $T M_{01}$ & $T M_{01}, T M_{02}$ & $T M_{21}, T M_{02}$ & $\begin{array}{l}\text { (multiple } \\
\text { modes) }\end{array}$ & $T M_{01}, T M_{02}$ \\
\hline $\begin{array}{l}\text { Substrate- } \\
\text { integrated }\end{array}$ & $\checkmark$ & - & $\checkmark$ & $\checkmark$ & $\checkmark$ & - & - & $\checkmark$ \\
\hline Frequency $(\mathrm{GHz})$ & 5.8 & $3-5.5$ & 5.9 & 5.8 & 2.26 & $1.7-2.5$ & $2.15-14$ & $5.05-6.65$ \\
\hline $\begin{array}{l}\text { Largest } \\
\text { dimension }(\mathrm{mm})\end{array}$ & $\begin{array}{c}23 \text { (diagonal) } \\
0.4 \cdot \lambda_{0}\end{array}$ & $\begin{array}{c}74 \text { (diagonal) } \\
0.7 \cdot \lambda_{0, \max } \\
\end{array}$ & $\begin{array}{c}62 \text { (diameter) } \\
1.2 \cdot \lambda_{0}\end{array}$ & $\begin{array}{c}40 \text { (diameter) } \\
0.8 \cdot \lambda_{0}\end{array}$ & $\begin{array}{c}67 \text { (diameter }) \\
0.5 \cdot \lambda_{0, \max }\end{array}$ & $\begin{array}{c}57 \text { (diagonal) } \\
0.3 \cdot \lambda_{0, \max }\end{array}$ & $\begin{array}{c}40 \text { (diameter) } \\
0.5 \cdot \lambda_{0, \max }\end{array}$ & $\begin{array}{c}64 \text { (diameter) } \\
1.1 \cdot \lambda_{0, \max }\end{array}$ \\
\hline $\begin{array}{l}\text { Substrate height } \\
(\mathrm{mm})\end{array}$ & $3.2\left(\varepsilon_{r}=2.1\right)$ & 8 (foam) & $\begin{array}{c}1.5 \\
\left(\varepsilon_{r}=2.94\right) \\
\end{array}$ & $\begin{array}{c}0.79 \\
\left(\varepsilon_{r}=2.33\right) \\
\end{array}$ & $\begin{array}{c}3.2 \\
\left(\varepsilon_{r}=2.33\right) \\
\end{array}$ & 12 (air) & 10 (air) & $1.5\left(\varepsilon_{r}=2.65\right)$ \\
\hline Bandwidth & $2.1 \%$ & $70 \%$ & $12.8 \%$ & $1.5 \%$ & $18 \%$ & $>50 \%$ & $147 \%$ & $27.4 \%$ \\
\hline
\end{tabular}

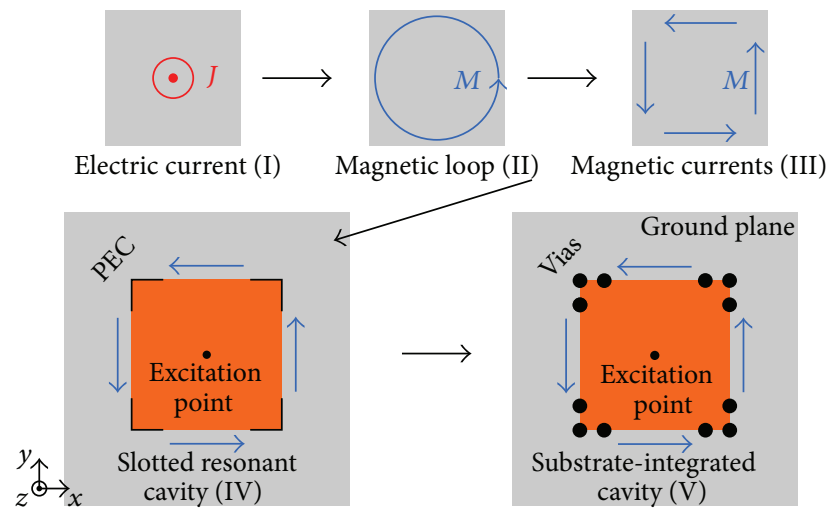

FIGURE 1: Radiation principle as an evolution from electric current perpendicular to a ground plane to a slotted substrate-integrated cavity.

The paper first introduces the radiation principle in the next section, followed by specific design guidelines in Section 3. An experimental validation in the DSRC band around $5.9 \mathrm{GHz}$ band validates the concept in Section 4 .

\section{Radiation Principle}

The proposed concept of low-profile antenna with omnidirectional monopole pattern is based on the illustrations in Figure 1. First, a monopole antenna based on a vertical electric current (I) on a ground plane is replaced with an equivalent magnetic current loop with constant distribution (II). This circular loop is approximated through four in-phase magnetic current elements (III). The spacing between the magnetic current elements must be less than half a free-space wavelength to avoid grating lobes. From the equivalence principle, it is well-known that magnetic currents can be realized from thin slots in a metallic cavity (IV). For the sake of design robustness to tolerances, monomode operation in the fundamental cavity mode is desirable, whereas the pure cavity mode is completely determined through the side walls and substrate properties. In the present case, the fundamental mode is not the mode of a purely metallic cavity, as it is disturbed along the openings in the side walls, where the apertures act as perfect magnetic conductors. It should be noted, however, that the equivalent magnetic walls are only a valid assumption if the substrate height is sufficiently small such that the electric fields are transverse to the aperture. As an interesting observation, these radiating apertures are similar to those of half-mode substrate-integrated cavities such as in $[9,11]$.

Nevertheless, the field distribution resembles a $T M_{11}$ mode of a square cavity. It is observed that the length of the four apertures influences the resonance frequency significantly but also affects the radiation efficiency as discussed in the next section. In the practical implementation, the structure can be realized through a substrate-integrated cavity (V) with via side walls forming the equivalent perfect electric conductor. The instantaneous electric field distribution for the fundamental resonance of the resulting structure is shown in Figure 2. Since this cavity is operating in the monomode region, the structure is robust in terms of manufacture tolerances and feed position accuracy, as no parasitic modes can be excited.

It should be noted that the operating principle of the proposed structure is not the same as that of a microstrip patch antenna due to the difference in the resonant cavity mode: rectangular microstrip patch antennas operate in their $T M_{01}$ resonance as two radiating slots, corresponding to two parallel magnetic currents with the same direction. In contrast, the proposed antenna operates with four equivalent current elements forming a near-constant magnetic current loop.

\section{Design}

The design is based on a Rogers Duroid 5880 substrate with a relative permittivity of $\varepsilon_{r}=2.2$ and a loss tangent of $\tan \delta=0.0009$. The thickness has been chosen as $h=3.2 \mathrm{~mm}$, which corresponds to $\lambda_{0} / 16$ at the targeted resonance frequency of $5.8875 \mathrm{GHz}$ in the DSRC band. The required bandwidth is $75 \mathrm{MHz}$, which corresponds to $1.3 \%$. An emphasis in the design is set on simple manufacture with a minimal amount of elements. Thus, a square design is selected because of its symmetry, with identical lengths, widths, and via positions. The feeding technique is a direct 


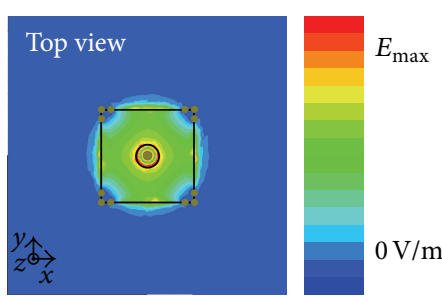

(a)

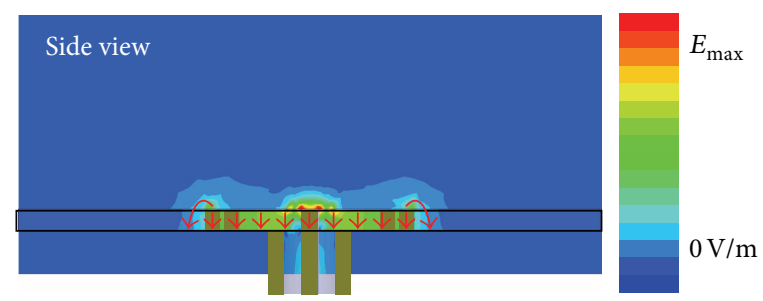

(b)

FIGURE 2: Instantaneous electric field distribution on resonance: (a) top view, (b) side view.

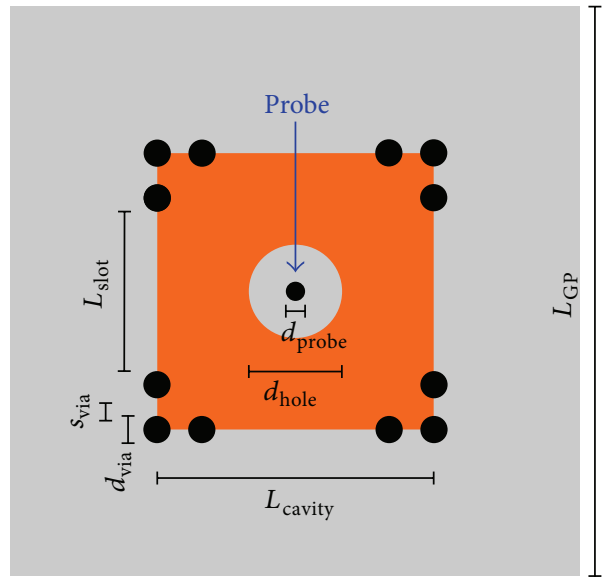

(a)

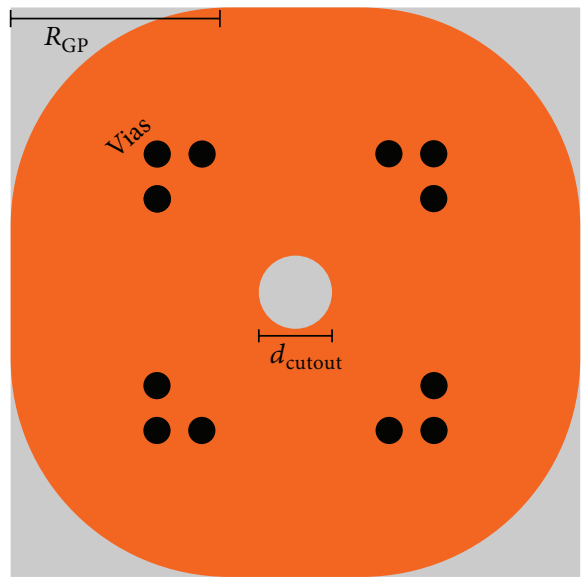

(b)

FIGURE 3: Geometry of the proposed substrate-integrated cavity monopole antenna: (a) top layer, (b) bottom layer.

TABLE 2: Dimensions of the antenna (mm).

\begin{tabular}{lcccccccc}
\hline$L_{\text {cavity }}$ & $d_{\text {via }}$ & $s_{\text {via }}$ & $L_{\text {slot }}$ & $d_{\text {probe }}$ & $d_{\text {hole }}$ & $L_{\mathrm{GP}}$ & $R_{\mathrm{GP}}$ & $d_{\text {cutout }}$ \\
\hline 16.0 & 1.05 & 1.35 & 10.2 & 1.27 & 6.17 & 55.0 & 25.0 & 4.1 \\
\hline
\end{tabular}

consequence of this simplicity requirement and is achieved through a probe-feed from a $50 \Omega$ SMA connector through the ground plane.

For a practical realization, each metallic wall cavity corner wall can be replaced with three vias with a diameter of $d_{\text {via }}$ and a spacing of $s_{\text {via }}$ as given in Table 2. The vias are manufactured through rivets attached and soldered after etching of the PCB. The size and spacing of the vias determine the aperture size, as discussed in the next section. In total, only twelve vias are required to maintain the fundamental $T M$-mode, which results in a low-cost printed circuit board. The chosen arrangement of vias allows control of the mode in the cavity, the matching, and the four aperture sizes and hence the radiation characteristics. Furthermore, it enforces the symmetry of the structure and improves the robustness to tolerances.

3.1. Cavity. The structure is shown in Figure 3, with the optimized dimensions given in Table 2. The resonance frequency is determined by the cavity size $L_{\text {cavity, }}$ the substrate relative permittivity $\varepsilon_{r}$, and the slot length $L_{\text {slot }}$, which is delimited by the equivalent via wall, that is, determined by the via diameter $d_{\text {via }}$ and spacing $s_{\text {via }}$. A larger cavity resonates at a lower frequency, with a reduced bandwidth for a fixed height [12]. The optimized cavity side length corresponds to $\lambda_{0} / 3$ at the resonance frequency, which satisfies the mentioned requirements that the equivalent magnetic current spacing has to be smaller than half a free-space wavelength. Higher permittivities of the substrate lower the resonance frequency for a given cavity size, but at a cost of higher losses and decreased bandwidth.

3.1.1. Slot Length. The slot length also determines the resonance frequency: the fringing of the fields is increased for larger apertures, which increases the effective size of the cavity and hence lowers the resonance frequency. This is shown in Figure 4, through simulation results obtained with a simplified model using a lumped port in the cavity center and perfect electric walls. It is noted that the resonance frequency for this cavity converges to the theoretically expected value for the $T M_{11}$ mode of a closed cavity when $L_{\text {slot }}$ is reduced to $0 \mathrm{~mm}$; that is,

$$
f_{r}=\frac{c}{\sqrt{2 \varepsilon_{r}} L_{\text {cavity }}}=8.93 \mathrm{GHz}
$$

Increasing the slot length $L_{\text {slot }}$ decreases the resonance frequency, reducing it to almost half at $L_{\text {slot }}=12.5 \mathrm{~mm}$ compared to the closed cavity. Furthermore, it is observed that if the radiating aperture along the aperture is short, the 


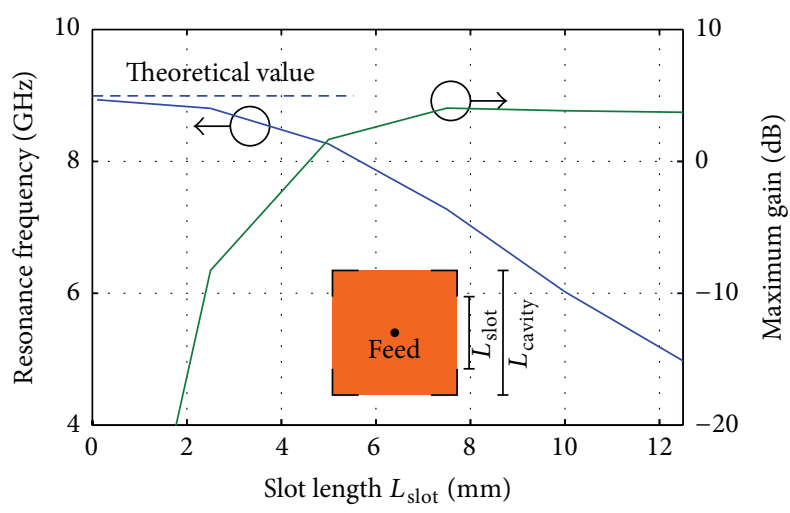

FIGURE 4: Influence of slot length on the resonance frequency and maximum gain of the simplified structure shown in the inset for $L_{\text {cavity }}=16 \mathrm{~mm}$.

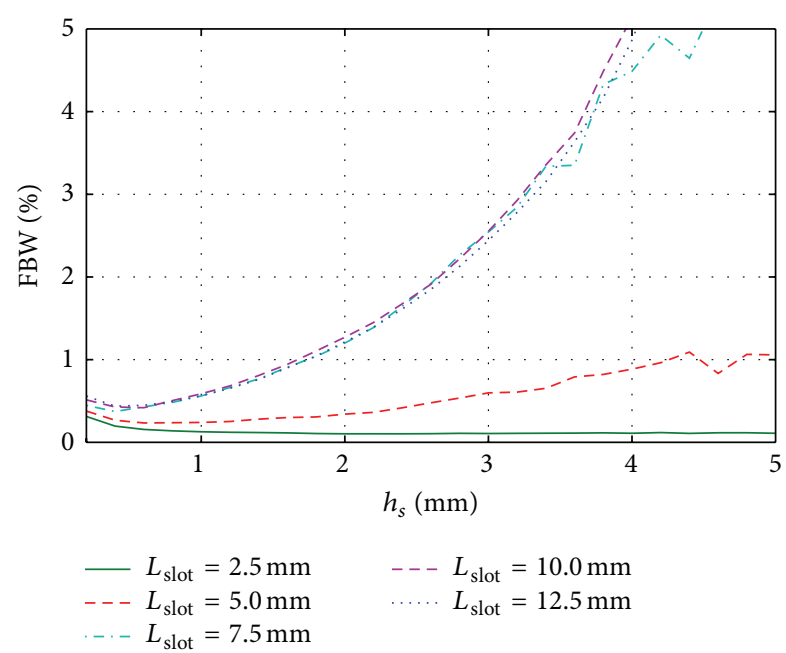

FIGURE 5: Dependence of the substrate height on the fractional bandwidth for various slot lengths.

radiation efficiency is very low. This is exemplified in Figure 4, where the maximum gain only becomes larger than $0 \mathrm{~dB}$ for a slot length larger than $L_{\text {slot }}=4.5 \mathrm{~mm}$.

3.1.2. Substrate Height. The impact of the substrate height on the fractional bandwidth for the simplified structure is shown in Figure 5. Given sufficiently large slots $\left(L_{\text {slot }}>7.5 \mathrm{~mm}\right.$ in this design), an almost identical behavior for different slot lengths can be observed: increasing the resonator height significantly increases the bandwidth. This behavior is consistent with that from related structures such as microstrip patch antennas.

3.2. Ground Plane. While the resonator shape and realization influence the impedance matching and radiation characteristics, the effects of the ground plane size and shape are mainly observed in the radiation pattern. Since the antenna near fields and hence the antenna matching are not significantly influenced by this effect, it can be treated as an independent design problem. In general, resonances of the ground plane can create ripples in the pattern and excite cross polarization components in the radiated fields. In order to minimize the cross polarization, the shape of the metallic ground plane has been modified to a rounded rectangle with a radius $R_{\mathrm{GP}}$ as shown in Figure 3. Numerical evaluations have shown that this radius should be chosen as large as possible to minimize cross polarization. The choice of the size $L_{\mathrm{GP}}$ is a trade-off: a larger ground plane helps in reducing the cross polarization but comes at a cost of a reduced broad-side gain. In this design, a radius of $R_{\mathrm{GP}}=25 \mathrm{~mm}$ and ground plane size of $L_{\mathrm{GP}}=55 \mathrm{~mm}$ have been chosen.

It should be noted that the mounting of the antenna for the proposed application will further influence the radiation pattern, for example, the placement in the metallic roof of a vehicle. Hence, this remains the object of further considerations.

3.3. Capacitive Ring Feed. Because the considered cavity is very shallow, the input impedance is very high, which is typical for cavity-backed magnetic loop antennas [13]. Simulation results show input resistances in the order of $1000 \Omega$. Hence, a good match requires additional efforts. In this case, a ring-loaded coaxial feed [14] is simplified to a feed probe with a coaxial gap as shown in Figure 3. In this implementation the probe traverses the substrate and its open end is surrounded by a hole of diameter $d_{\text {hole }}$ on the top metalization. Due to the capacitance of this gap, the input impedance can be controlled without additional elements in the antenna. An alternative approach is a capacitive gap filled with high-permittivity material between the top plane and the probe, as presented in [15], with the advantage of increased bandwidth.

The gap size has two main influences on the antenna performance. As a primary effect shown in Figure 6, the magnitude of the input impedance of the antenna decreases for increased capacitive gap sizes $d_{\text {hole }}$. The input resistance $\operatorname{Re}(Z)$ starts from $550 \Omega$ at a capacitive gap size of $d_{\text {hole }}=$ $1.4 \mathrm{~mm}$ and decreases to $29 \Omega$ at $d_{\text {hole }}=11.3 \mathrm{~mm}$. The input reactance has a negative value which also decreases for increasing gap sizes. As the capacitive ring feed changes the shape of the resonant cavity, the resonance frequency is also influenced as a secondary effect. As shown in Figure 7, increasing the cap size increases the resonance frequency from $5.2 \mathrm{GHz}$ to $6.6 \mathrm{GHz}$ within the considered range of capacitive gap sizes from $d_{\text {hole }}=1.4 \mathrm{~mm}$ to $11.3 \mathrm{~mm}$.

The gap size and antenna aperture size have been optimized for matched resistance of $50 \Omega$ at a resonance frequency of $5.9 \mathrm{GHz}$, resulting in the hole diameter given in Table 2. The resulting simulated reflection coefficient of the structure is shown in Figure 9, which shows a good match within the DSRC band indicated in the figure. The instantaneous electric field distribution around the coaxial capacitive gap is visible in Figure 2. A strong electric field is observed, which can contribute to substrate losses in the antenna. Due to the small size of the gap, no significant power is radiated from that region and the overall radiation pattern is not influenced by the feed. 


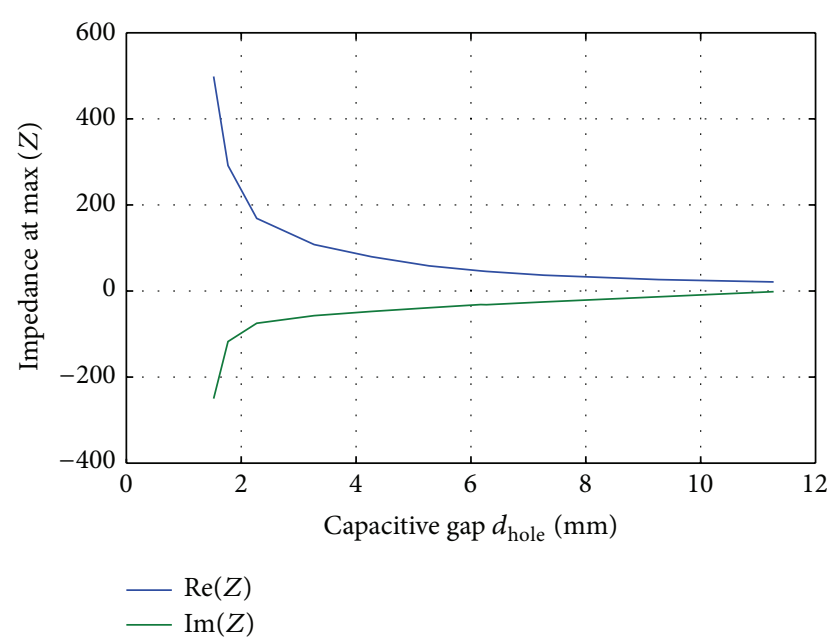

FIGURE 6: Impact of capacitive gap $d_{\text {hole }}$ on the input impedance of the antenna.

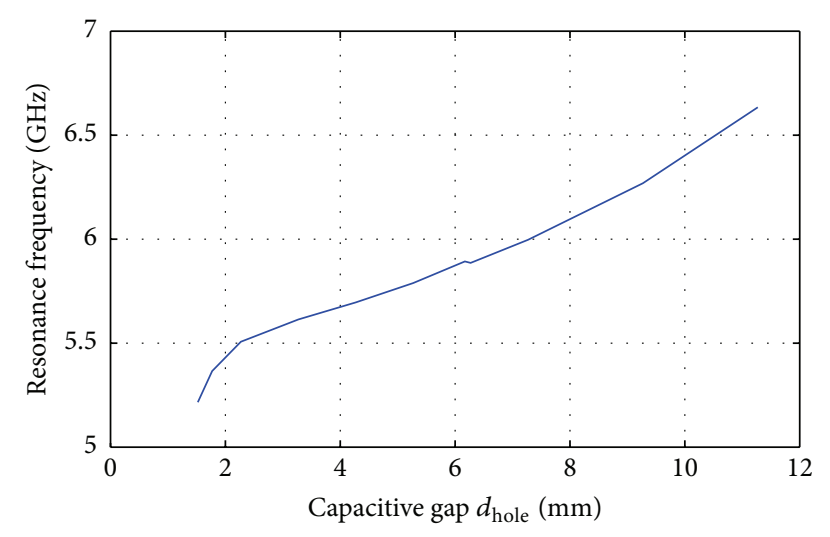

FIGURE 7: Impact of capacitive gap $d_{\text {hole }}$ on the resonance frequency of the antenna.

\section{Measurement}

A prototype of the antenna has been manufactured and is shown in Figure 8. Due to the simple manufacture method which requires only simple patterning and a few elements, a first-pass successful design is likely. The measured reflection coefficient is shown in Figure 9. A deep resonance at $5.86 \mathrm{GHz}$ is observed with only a slight frequency shift compared to the simulation results. The measured bandwidth is $130 \mathrm{MHz}$, which corresponds to around $2.1 \%$ fractional bandwidth. The measured radiation patterns are shown in Figure 10. The expected monopole radiation pattern is observed with an omnidirectional pattern in the $x y$-plane and a sinusoidal angular dependence in the elevation planes. The cross polarization in the $x y$-plane exhibits a four-leave clover shape, which is expected due to the asymmetry of the square cavity. The measured maximum gain of $0.5 \mathrm{~dB}$ is slightly lower compared to other reported results [3-5], which is attributed to the small size of the antenna and fabrication imperfections.
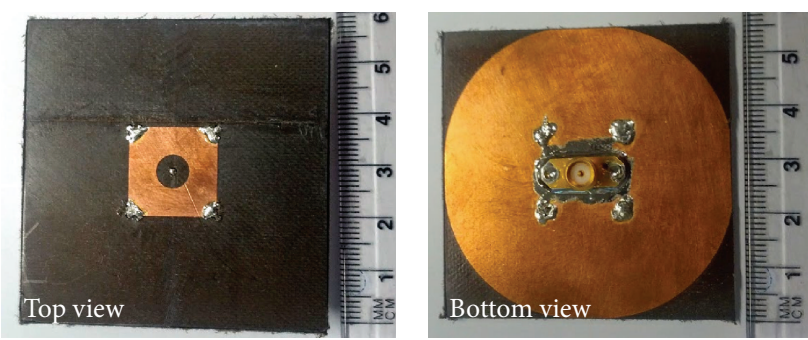

FIgURE 8: Manufactured prototype based on Rogers Duroid 5880 substrate.

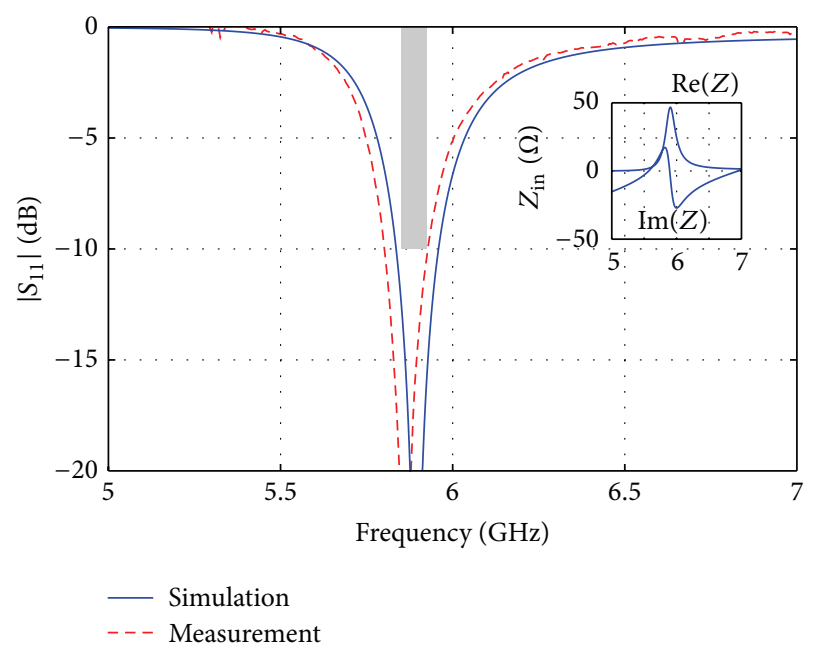

FIGURE 9: Simulated and measured reflection coefficient of the antenna. The simulated input impedance is given in an inset.

\section{Conclusion}

A low-profile cavity-backed planar monopole antenna has been introduced for the DSRC band around $5.9 \mathrm{GHz}$, which has potential applications in vehicle-to-vehicle communications. The structure is based on a square substrateintegrated cavity with shorting vias in each corner operating in fundamental $T M_{11}$ mode. This new robust design based on a cavity resonator offers a new design paradigm for substrate-integrated monopole antennas and exhibits competitive overall performance in a compact and simple realization (Table 1). Four apertures along the cavity side walls form four equivalent in-phase magnetic currents which act as a magnetic current loop antenna, with omnidirectional radiation equivalent to a monopole antenna. The antenna is fed through an open SMA probe with a coaxial capacitive gap for matching. The reflection coefficient and radiation patterns measured for a manufactured prototype operating at $5.9 \mathrm{GHz}$ correspond well with the simulation results with an impedance bandwidth of $2.1 \%$ generously covering the DSRC band and omnidirectional patterns with low cross polarization. This makes this design a good candidate for vehicle-to-vehicle communication systems. Further potential applications include wearable systems for on- and off-body communication. For other applications, the bandwidth can 


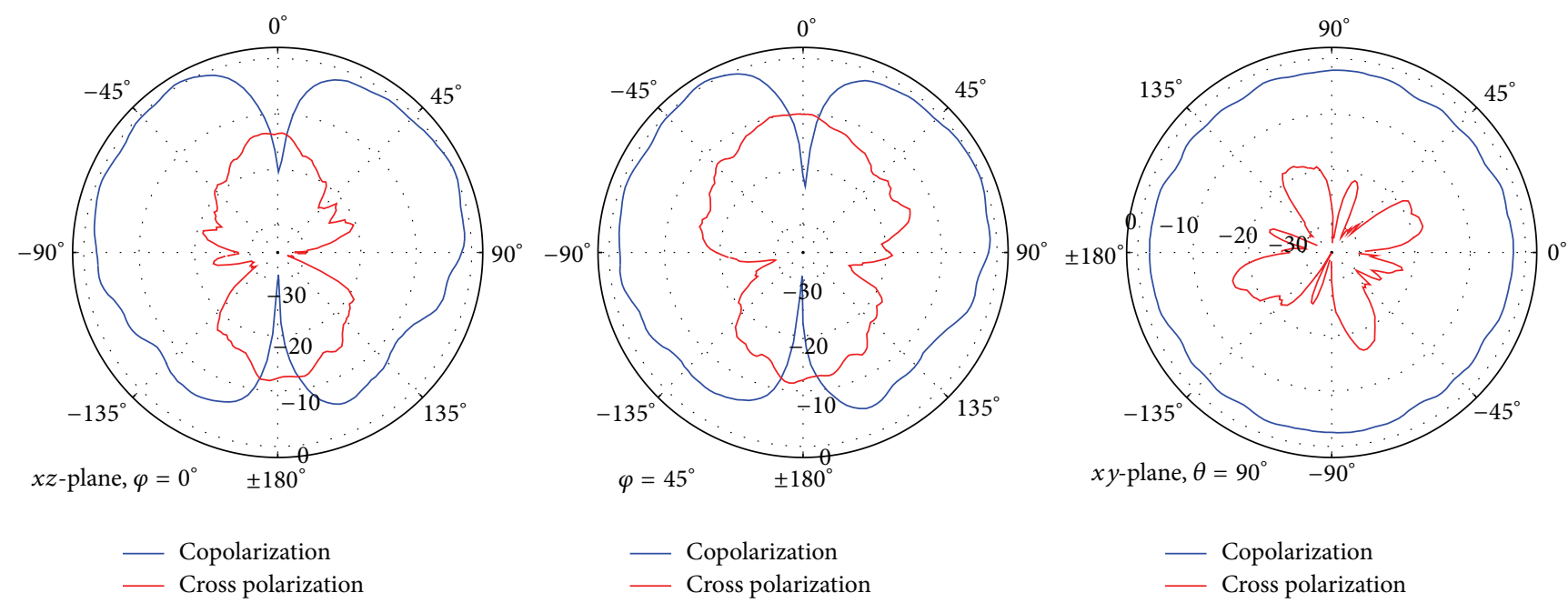

FIGURE 10: Measured realized gain pattern along the $x z$-plane, a plane inclined by $\varphi=45^{\circ}$, and the omnidirectional $x y$-plane.

be increased by increasing the substrate height or reducing the substrate permittivity. In a next step, other feeding techniques such as aperture coupling will be evaluated for a direct integration of the antenna into a printed circuit board.

\section{Conflict of Interests}

The authors declare that there is no conflict of interests regarding the publication of this paper.

\section{Acknowledgment}

C. Fumeaux acknowledges the support of the Australian Research Council (ARC) Future Fellowship funding scheme (under FT100100585).

\section{References}

[1] S. Biswas, R. Tatchikou, and F. Dion, "Vehicle-to-vehicle wireless communication protocols for enhancing highway traffic safety," IEEE Communications Magazine, vol. 44, no. 1, pp. 7482, 2006.

[2] S.-J. Lin and J.-S. Row, "Monopolar patch antenna with dualband and wideband operations," IEEE Transactions on Antennas and Propagation, vol. 56, no. 3, pp. 900-903, 2008.

[3] A. Al-Zoubi, F. Yang, and A. Kishk, "A broadband center-fed circular patch-ring antenna with a monopole like radiation pattern," IEEE Transactions on Antennas and Propagation, vol. 57, no. 3, pp. 789-792, 2009.

[4] L. Economou and R. J. Langley, "Patch antenna equivalent to simple monopole," Electronics Letters, vol. 33, no. 9, pp. 727-729, 1997.

[5] J. Liu, Q. Xue, H. Wong, H. W. Lai, and Y. Long, "Design and analysis of a low-profile and broadband microstrip monopolar patch antenna," IEEE Transactions on Antennas and Propagation, vol. 61, no. 1, pp. 11-18, 2013.

[6] J.-S. Row and S.-H. Chen, "Wideband monopolar square-ring patch antenna," IEEE Transactions on Antennas and Propagation, vol. 54, no. 4, pp. 1335-1339, 2006.
[7] H. Nakano, H. Iwaoka, K. Morishita, and J. Yamauchi, "A wideband low-profile antenna composed of a conducting body of revolution and a shorted parasitic ring," IEEE Transactions on Antennas and Propagation, vol. 56, no. 4, pp. 1187-1192, 2008.

[8] J. Liu, S. Zheng, Y. Li, and Y. Long, "Broadband monopolar microstrip patch antenna with shorting vias and coupled ring," IEEE Antennas and Wireless Propagation Letters, vol. 13, pp. 3942, 2014.

[9] S. A. Razavi and M. H. Neshati, "Development of a low-profile circularly polarized cavity-backed antenna using HMSIW technique," IEEE Transactions on Antennas and Propagation, vol. 61, no. 3, pp. 1041-1047, 2013.

[10] M. Esquius-Morote, B. Fuchs, J.-F. Zurcher, and J. R. Mosig, "Novel thin and compact H-plane SIW horn antenna," IEEE Transactions on Antennas and Propagation, vol. 61, no. 6, pp. 2911-2920, 2013.

[11] T. Kaufmann and C. Fumeaux, "Wearable textile half-mode substrate-integrated cavity antenna using embroidered vias," IEEE Antennas and Wireless Propagation Letters, vol. 12, pp. 805-808, 2013.

[12] C. A. Balanis, Antenna Theory: Analysis and Design, WileyInterscience, 2005.

[13] C. R. Cockrell, "The input admittance of the rectangular cavitybacked slot antenna," IEEE Transactions on Antennas and Propagation, vol. 24, no. 3, pp. 288-294, 1976.

[14] E. Arnieri, G. Amendola, L. Boccia, and G. Di Massa, "Coaxially fed substrate integrated radiating waveguides," in Proceedings of the IEEE Antennas and Propagation Society International Symposium (AP-S '07), pp. 2718-2721, Honolulu, Hawaii, USA, June 2007.

[15] N. Nguyen-Trong, T. Kaufmann, and C. Fumeaux, "Wideband transition from coaxial line to half-mode substrate integrated waveguide," in Proceedings of the 3rd Asia-Pacific Microwave Conference (APMC '13), pp. 110-112, November 2013. 

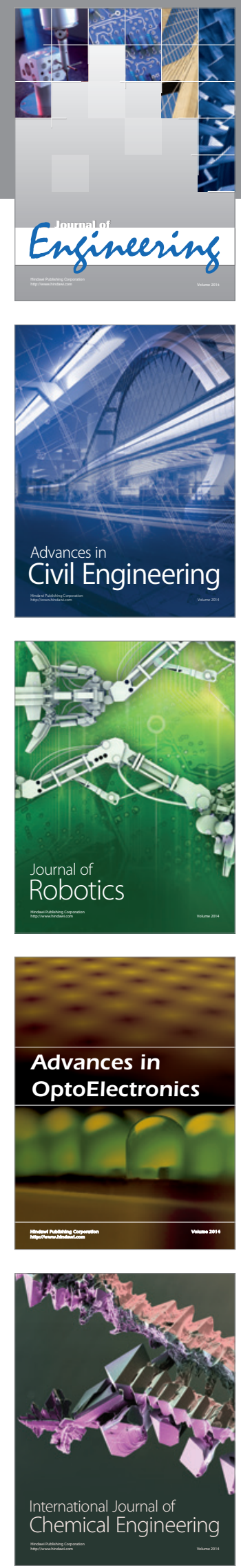

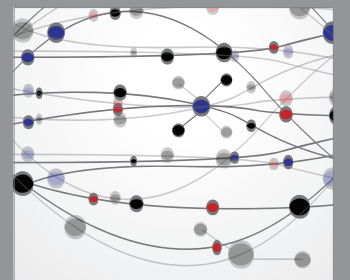

The Scientific World Journal
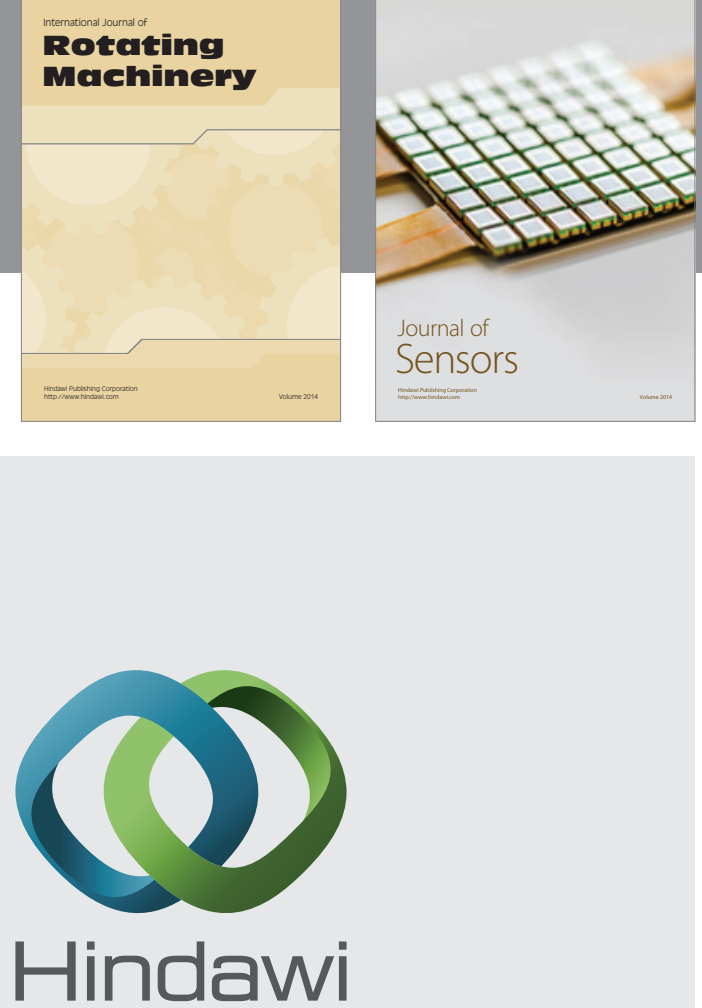

Submit your manuscripts at http://www.hindawi.com
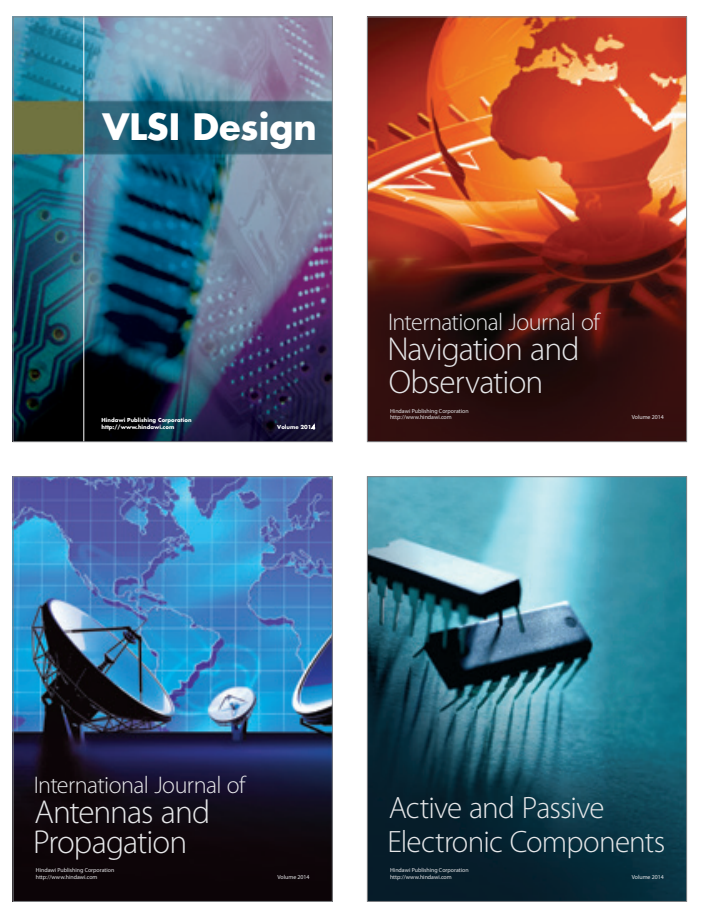
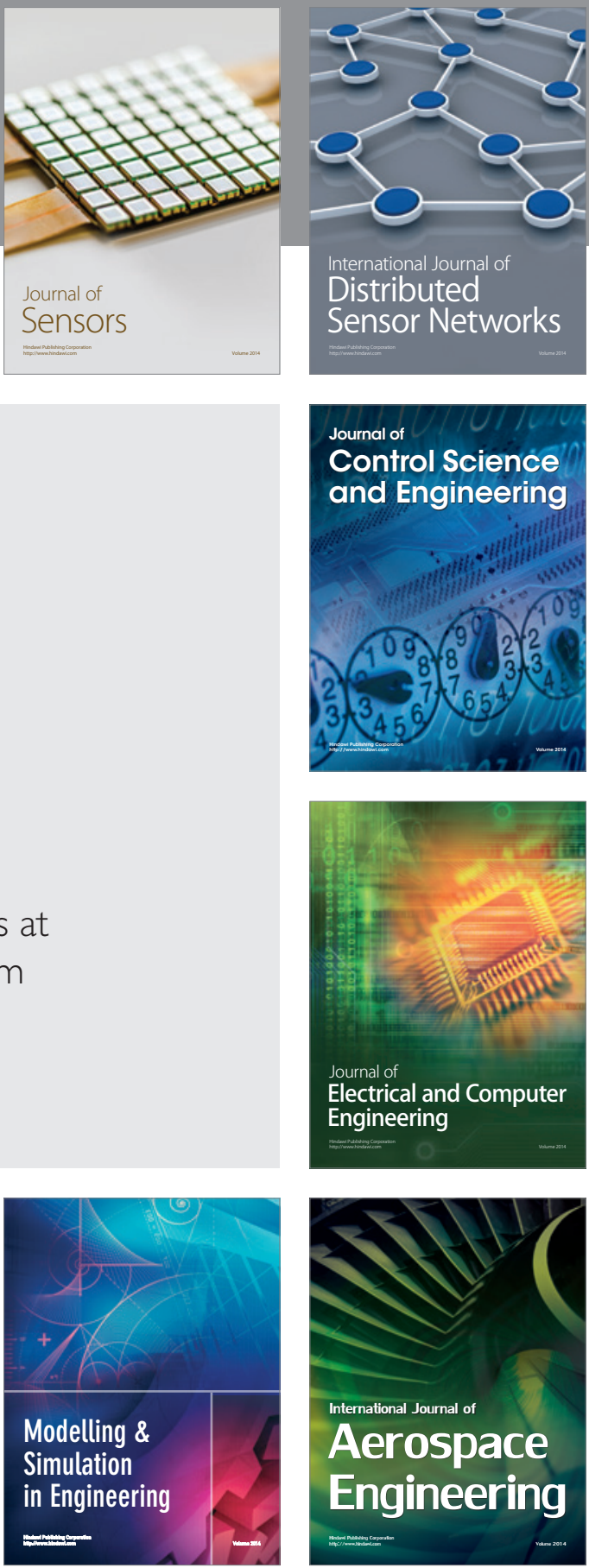

Journal of

Control Science

and Engineering
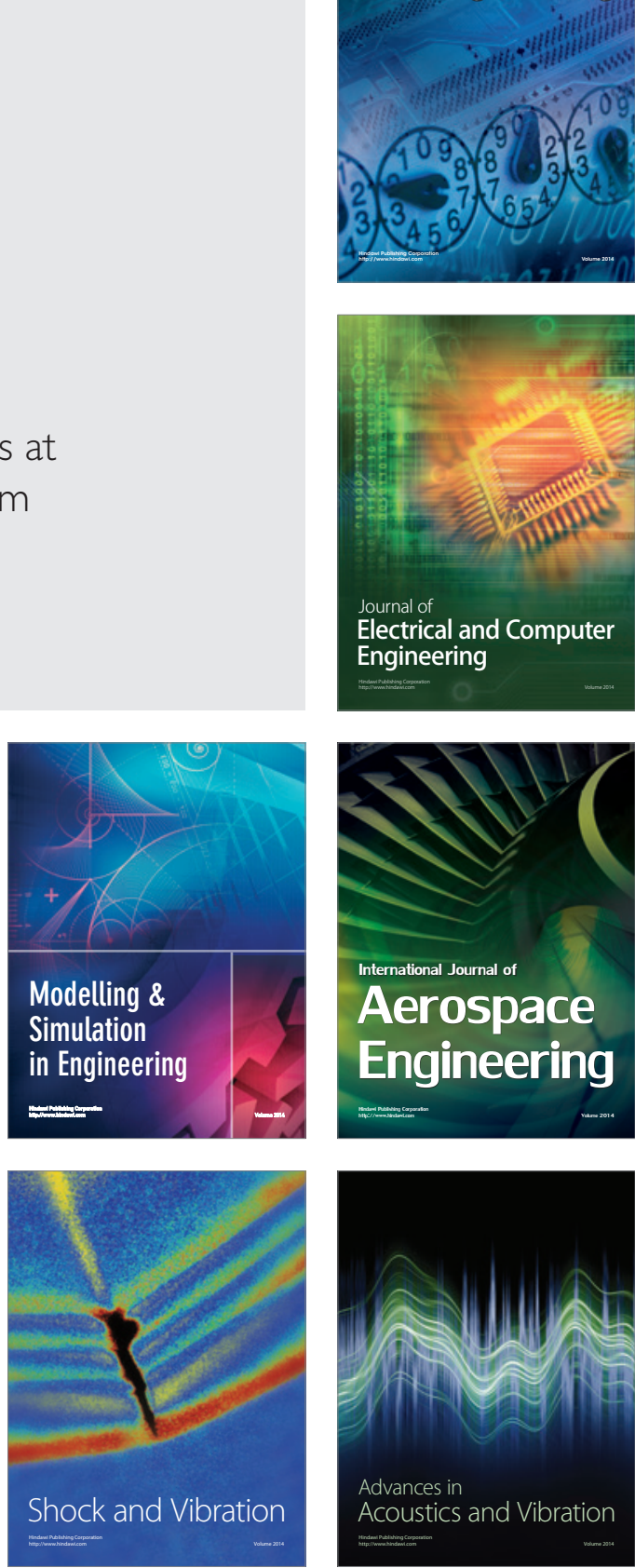